\title{
From the unification scale to the weak scale: A self consistent supersymmetric Dine-Fischler-Srednicki-Zhitnitsky axion model
}

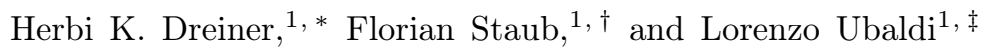 \\ ${ }^{1}$ Bethe Center for Theoretical Physics $\&$ Physikalisches Institut der Universität Bonn, \\ 53115 Bonn, Germany
}

\begin{abstract}
The distinguishing feature of the Dine-Fischler-Srednicki-Zhitnitsky (DFSZ) axion is that it couples to the electroweak Higgs fields. There is thus an immediate connection between the PecceiQuinn (PQ) scale and the weak scale. We wish to incorporate the DFSZ axion in a complete supersymmetric model, valid at all scales, and then to implement it in a numerical code connecting the high scale and the low scale physics on a quantitative level. We find that the simplest supersymmetric DFSZ model, as proposed by Rajagopal et al. in 1990, is inconsistent when we consider the minimization of the scalar potential. The problem is that we obtain a negative squared mass for the saxion, the scalar partner of the axion, at the minimum. We then consider the minimal extension in order to get a consistent model for all scales: one has to include an additional explicit sector to spontaneously break the PQ symmetry. In the complete model we can determine the mass of the axino, the fermionic partner of the axion. It is useful to distinguish two cases: (1) the supersymmetry (SUSY) breaking scale is lower than the PQ breaking scale, and (2) the scales are comparable. We find that the axino is very light in (1), while its mass is generically of the order of the other soft SUSY breaking masses in (2). We have implemented SUSY breaking via generic soft breaking terms, and thus make no explicit statement about the form and mediation of SUSY breaking. This complete model can then be incorporated in a numerical code connecting the two scales. We briefly discuss the renormalization group equations and the couplings of the axion to gluons and photons.
\end{abstract}

\section{INTRODUCTION}

The Minimal Supersymmetric Standard Model (MSSM) [1 is a complete model, which in principle should explain all phenomena from low-energy physics up to possible observations at the $\mathrm{TeV}$ scale. In its full version it contains over a 100 free parameters. To make it more predictive one typically makes simplifying assumptions at the unification scale reducing the number of parameters to only 5 . This is for example the case in the constrained minimal supersymmetric model (CMSSM) 2]. The strength of such a model is that one can test it in a self consistent way against basically all experimental data. This includes a vast number of experiments in a wide energy range, for example measurements of the anomalous magnetic moment of the muon, LHC data, as well as direct and indirect searches for dark matter. This is possible thanks to the renormalization group equations (RGEs) which relate parameters at different energy scales [3, 4]. Because of its simplicity and its predictive power the CMSSM has been widely studied for years. Due to the recent strict lower mass bounds on superpartner masses found at the LHC the CMSSM is in some tension with the Higgs mass measurement, as well as fine-tuning requirements [5, 6].

A theoretical problem which is left unaddressed in the MSSM and in the CMSSM is the strong CP problem [7]. The most plausible solution is provided by the axion [8, 9], which can be implemented in two ways. In the first, the axion couples to new heavy quarks [10, 11] charged under the color $S U(3)$ gauge group and the global Peccei-Quinn (PQ) $U(1)_{\mathrm{PQ}}$ symmetry [12. Models of this kind are referred to as KSVZ. Here the axion sector is decoupled from the low-energy fields and we do not consider these models any further. In the second implementation, the DFSZ model [13, 14, the axion couples to two electroweak Higgs-doublet fields, which are charged under the $U(1)_{\mathrm{PQ}}$; the Standard Model fermions then also carry PQ charge. Employing the axion solution in a supersymmetric model requires promoting the axion to a superfield, which introduces two other new particles: the saxion, a scalar, and the axino, a fermion. The axion and also the axino have the virtue of themselves being good dark matter candidates [13, 15].

Indeed the axino as a dark matter candidate has been widely explored. However the studies in the literature [16 22] are not based on a complete model. Instead they consider the axion/axino sector separately, represented by only a few operators, often in an effective field theory framework, which encode the interactions of matter with the axino relevant only to its cosmological and thermal history. In that way one can get constraints that are fairly model independent. However with this approach it is difficult to correlate constraints coming from different observables at different energies, in particular also relating to the electroweak sector. If one wanted to do so then one would have to refer to a specific more complete model.

A step in the direction of a phenomenological axion model in supersymmetry (SUSY) to study constraints in a fashion similar to the CMSSM, was taken by Baer and collaborators 2328 . In particular in a recent paper [29] they study a SUSY DFSZ axion model. 
Their starting point consists of two different benchmark models initially without the axion: one is denoted "radiatively-driven natural SUSY" [30, the other is the minimal supergravity model (mSUGRA). They first generate the (non-axion/axino) SUSY model mass spectra with ISAJET 31 for both models, and only as a second step, after running the RGEs, they add the axion superfield with its interactions and study the resulting phenomenology. Thus these interactions are treated separately and do not enter in the details of the numerical calculations of the spectrum generator. This is somewhat justified by the fact that the interactions of the axion supermultiplet with matter are suppressed by inverse powers of the high PQ scale, $f_{a} \sim 10^{12} \mathrm{GeV}$. However we believe that it would be desirable to go a step further, and include the axion from the start and study the full model consistently. By using the same boundary conditions at the GUT or SUSY breaking scale for the soft parameters in the axion/axino sector as for the MSSM the model will become more predictive. This should also be particularly relevant when extending the model to R-parity violating supersymmetry, where the axino also mixes with the neutrinos and neutralinos [17.

The aim of the current paper is to study what are the minimal ingredients and assumptions needed to define a self consistent SUSY axion model, in order to later study its phenomenological implications with numerical tools available today. We concentrate on a DFSZ, R-parity conserving SUSY model. As SUSY already requires two electroweak Higgs doublets, this model is more economical than its KSVZ counterpart. The minimal model one might first consider is the one introduced in Ref. [15]. We show that this model has an inconsistent scalar sector: the saxion becomes tachyonic. We are thus forced to add superpotential terms that spontaneously break the PQ symmetry. The resulting model is self consistent and predictive. One prediction, for example, is that the mass of the heavy Higgs is modified compared to the MSSM.

In the context of these models we give what we consider a sharper and more intuitive answer to a question which has been investigated in the literature [32 34]: what is the axino mass, $m_{\tilde{a}}$, with broken supersymmetry? We consider two cases. In the first and simpler case, the SUSY breaking scale, $M_{\mathrm{SB}}$, is much smaller than the PQ breaking scale, $f_{a}$. Then we find that $m_{\tilde{a}} \sim \mathcal{O}\left(\frac{M_{\mathrm{SUSY}}^{2}}{f_{a}}\right)$, with $M_{\mathrm{SUSY}} \sim \mathrm{TeV}$ the scale of the soft SUSY breaking terms. So the axino is very light. In the second case, we consider $M_{\mathrm{SB}} \geq f_{a}$, which is representative of gravity mediated SUSY breaking models where typically $M_{\mathrm{SB}} \sim$ $M_{\mathrm{GUT}}$, and find that $m_{\tilde{a}} \sim M_{\mathrm{SUSY}}$. Our computations agree with previous claims while providing a hopefully new and edifying perspective on the issue.

\section{THE MINIMAL INCONSISTENT MODEL}

It is not straightforward to embed the DFSZ axion in supersymmetric models. The trouble is that the nonsupersymmetric DFSZ model [13, 14, contains a term $g \varphi^{2} H_{u}^{\dagger} H_{d}$ in the scalar potential, where the phase of the complex scalar field $\varphi$ is the axion, $H_{u, d}$ are two complex Higgs doublets, and $g$ is a dimensionless real coupling constant. In supersymmetry this term can not be obtained from a renormalizable superpotential. To circumvent this difficulty Rajagopal et al. proposed [15] a recipe which consists in replacing the term $\mu \hat{H}_{u} \hat{H}_{d}$ in the superpotential (the hat here denotes a superfield) with the term

$$
c_{1} \hat{A} \hat{H}_{u} \hat{H}_{d}
$$

where $\hat{A}$ is the axion superfield and $c_{1}$ is a dimensionless constant. Once the scalar component of the superfield, $A$, gets a vacuum expectation value $(\mathrm{VEV})\langle A\rangle \sim f_{a}$, the parameter $\mu_{\mathrm{eff}}$ is given by $c_{1} f_{a}$. This implies that $c_{1}$ has to be tuned to $10^{-9}$ or $10^{-10}$ in order to get $\mu_{\text {eff }}$ at the weak scale. Despite being not a nice feature, this is no worse than the tuning which is needed for the coupling $g \leq 10^{-9}$ in the original DFSZ model [13], as already observed in Ref. [15].

A more elegant implementation of the DFSZ axion in SUSY can be obtained by dropping the requirement of renormalizability and writing a higher dimensional operator of the form $g \frac{\hat{A}^{2}}{M_{\mathrm{Pl}}} \hat{H}_{u} \hat{H}_{d}$, where $M_{\mathrm{Pl}}$ is the Planck mass. With a VEV $\langle A\rangle \sim 10^{11} \mathrm{GeV}$, this results in a $\mu_{\text {eff }}$ at the correct scale and thus provides a solution to the $\mu$-problem 35.

Nevertheless a renormalizable operator is easier to implement in a numerical study of the complete model, which is our main goal. Thus we choose the operator of Eq. (1) and accept the tuning of $\mu_{\text {eff }}$ for the time being. A further justification for the choice of this operator comes from a recent study [36], where the authors have derived a consistent realization of a SUSY DFSZ axion model within String Theory which can provide a microscopic explanation for the simple recipe of Rajagopal et al.

Let us then consider the model defined by the MSSM with the $\mu$-term replaced by Eq. (1). The full superpotential, assuming R-parity conservation, reads

$$
W=Y_{u} \hat{U} \hat{Q} \hat{H}_{u}+Y_{d} \hat{D} \hat{Q} \hat{H}_{d}+Y_{e} \hat{E} \hat{L} \hat{H}_{d}+c_{1} \hat{A} \hat{H}_{u} \hat{H}_{d} \text {. }
$$

$Y_{u, d, e}$ are $3 \times 3$ Yukawa matrices. We have suppressed generation and $S U(2)$ and $S U(3)$ gauge indices. The superfield $\hat{A}$ and the Standard Model superfields carry PQ charges, as is distinctive of DFSZ axion models, such that each term is invariant under the global $U(1)_{\mathrm{PQ}}$. We will see that the physical axion is a linear combination of the CP odd scalar components of $\hat{A}, \hat{H}_{u}$ and $\hat{H}_{d}$, in complete analogy with the non-SUSY model of Ref. [13. In order 
to compute the physical spectrum, one assumes that $A$ gets a vacuum expectation value $(\mathrm{VEV})\langle A\rangle \sim f_{a}$. We show that simply assuming such a VEV without specifying explicitly the $\mathrm{PQ}$ breaking mechanism and stabilizing the PQ potential leads to an inconsistency in the model.

The scalar potential is given by

$$
V=V_{F}+V_{D}+V_{\text {soft }}
$$

where

$$
\begin{aligned}
V_{F}= & \sum_{\phi}\left|\frac{\partial \widetilde{W}(\phi)}{\partial \phi}\right|^{2} \\
V_{D}= & \frac{1}{2} g_{i}^{2}\left(\Phi T_{\Phi}^{i, a} \Phi^{*}\right)\left(\Phi T_{\Phi}^{i, a} \Phi^{*}\right) \\
V_{\mathrm{soft}}= & T_{u} \tilde{u} \tilde{q} H_{u}+T_{d} \tilde{d} \tilde{q} H_{d}+T_{e} \tilde{e} \tilde{l} H_{d}+T_{c 1} A H_{u} H_{d} \\
& +m_{a}^{2}|A|^{2}+m_{H_{u}}^{2}\left|H_{u}\right|^{2}+m_{H_{d}}^{2}\left|H_{d}\right|^{2}+\tilde{\phi}^{\dagger} m_{\tilde{\phi}}^{2} \tilde{\phi}
\end{aligned}
$$

with $\tilde{\phi} \ni\{\tilde{e}, \tilde{l}, \tilde{d}, \tilde{u}, \tilde{q}\}$, as well as $A, H_{u}, H_{d}$ the scalar components of the respective superfields. Here $\widetilde{W}(\phi)$ denotes the superpotential evaluated as a function of scalar fields. $T_{u, d, e, c 1}$ are the trilinear soft breaking terms [37, elsewhere often denoted $A . T_{\Phi}^{i, a}$ are the gauge generators. To avoid clutter we take the soft parameters to be real in the following equations. This restriction does not affect our conclusions. The conventional $B \mu$-term resulting from Eq. (6) is given by $B_{\text {eff }}=T_{c 1}\langle A\rangle$.

The parameters have to fulfill the following tadpole equations to minimize the scalar potential at tree-level

$$
\begin{aligned}
\left.\frac{\partial V}{\partial \phi_{d}}\right|_{\phi=\sigma=0}= & m_{H_{d}}^{2} v_{d}+\frac{1}{8}\left[4 c_{1}^{2} v_{d}\left(v_{u}^{2}+f_{a}^{2}\right)-4 \sqrt{2} v_{u} B_{\mathrm{eff}}\right. \\
& \left.+v_{d}\left(g_{1}^{2}+g_{2}^{2}\right)\left(v_{d}^{2}-v_{u}^{2}\right)\right]=0 \\
\left.\frac{\partial V}{\partial \phi_{u}}\right|_{\phi=\sigma=0}= & m_{H_{u}}^{2} v_{u}+\frac{1}{8}\left[4 c_{1}^{2} v_{u}\left(v_{d}^{2}+f_{a}^{2}\right)-4 \sqrt{2} v_{d} B_{\mathrm{eff}}\right. \\
& \left.-v_{u}\left(g_{1}^{2}+g_{2}^{2}\right)\left(v_{d}^{2}-v_{u}^{2}\right)\right]=0 \\
\left.\frac{\partial V}{\partial \phi_{a}}\right|_{\phi=\sigma=0} & f_{a} m_{a}^{2}+\frac{1}{2 f_{a}}\left[\mu_{\mathrm{eff}}^{2}\left(v_{d}^{2}+v_{u}^{2}\right)\right. \\
& \left.-\sqrt{2} v_{d} v_{u} B_{\mathrm{eff}}\right]=0 .
\end{aligned}
$$

We have parametrized the scalar fields as in Ref. [13]:

$$
\begin{gathered}
H_{d}=\frac{1}{\sqrt{2}}\left(\phi_{d}+i \sigma_{d}+v_{d}\right), \quad H_{u}=\frac{1}{\sqrt{2}}\left(\phi_{u}+i \sigma_{u}+v_{u}\right) \\
A=\frac{1}{\sqrt{2}}\left(\phi_{a}+i \sigma_{a}+f_{a}\right) .
\end{gathered}
$$

The derivatives in Eqs. (7)-(9) are evaluated at the minimum, where $\phi_{d, u, a}=\sigma_{d, u, a}=0$. All tadpole equations and mass matrices have been calculated with the public code SARAH [38 42].

Upon closer examination, Eq. (9) presents a problem. In order to solve the hierarchy problem, the scale of the soft SUSY breaking terms [54], $M_{\mathrm{SUSY}}$, should be of order $M_{W}$. One would expect that $m_{a} \sim M_{\mathrm{SUSY}}$. For proper electroweak symmetry breaking, we must also have $\mu_{\text {eff }}^{2}, B_{\text {eff }}=\mathcal{O}\left(M_{W}^{2}\right)$. Under these conditions Eq. (9) is not soluble. Let us then fix $\mu_{\text {eff }}^{2}$ and $B_{\text {eff }}$ at $M_{\text {SUSY }}^{2}$ and solve for $m_{a}$. Then $m_{a} \sim M_{\mathrm{SUSY}}^{2} / f_{a}$ is tiny. This has an important consequence: it leads to a negative squared mass eigenvalue for the scalar field that we can identify as the saxion.

Before we show this let us briefly compute the CP odd scalar sector. After replacing the soft mass terms with the solutions of the tadpole equations the mass matrix squared in the basis $\left(\sigma_{d}, \sigma_{u}, \sigma_{a}\right)$ reads in the Landau gauge

$$
\mathcal{M}_{\text {CP odd }}^{2}=\left(\begin{array}{ccc}
B_{\text {eff }} t_{\beta} & B_{\text {eff }} & \frac{B_{\text {eff }} t_{\beta} v}{\sqrt{t_{\beta}^{2}+1} f_{a}} \\
B_{\text {eff }} & \frac{B_{\text {eff }}}{t_{\beta}} & \frac{B_{\text {eff }} v}{\sqrt{t_{\beta}^{2}+1} f_{a}} \\
\frac{B_{\text {eff }} t_{\beta} v}{\sqrt{t_{\beta}^{2}+1} f_{a}} & \frac{B_{\text {eff }} v}{\sqrt{t_{\beta}^{2}+1} f_{a}} & \frac{B_{\text {eff }} t_{\beta} v^{2}}{\left(t_{\beta}^{2}+1\right) f_{a}^{2}}
\end{array}\right) .
$$

Here we have written $t_{\beta} \equiv \tan \beta \equiv \frac{v_{u}}{v_{d}}$ for the ratio of the vacuum expectation values. The matrix Eq. (11) has two eigenvalues which are exactly zero. One is associated with the Goldstone boson which gets absorbed by the massive $Z$ boson. The other is associated with the axion, the Goldstone boson of the spontaneously broken (global) PQ symmetry. This represents a check that the SUSY breaking effects have not spoiled the Goldstone theorem [43]. The third eigenvalue is the mass squared of the physical CP odd Higgs boson

$$
m_{A}^{2}=B_{\mathrm{eff}}\left[t_{\beta}+\frac{1}{t_{\beta}}+\frac{t_{\beta} v^{2}}{\left(t_{\beta}^{2}+1\right) f_{a}^{2}}\right],
$$

which is the same as in the MSSM, apart from the very small correction given by the last term.

Let us turn now to the scalar mass matrix squared for the $\mathrm{CP}$ even states. After rotating the Higgs fields $\left(\phi_{d}, \phi_{u}\right) \rightarrow(h, H)$, it reads in the basis $\left(h, H, \phi_{a}\right)$ 


$$
\mathcal{M}_{\mathrm{CP} \text { even }}^{2}=\left(\begin{array}{ccc}
\frac{\left[16 \mu_{\mathrm{eff}}^{2} t_{\beta}^{2}+f_{a}^{2}\left(g_{1}^{2}+g_{2}^{2}\right)\left(t_{\beta}^{2}-1\right)^{2}\right] v^{2}}{4 f_{a}^{2}\left(t_{\beta}^{2}+1\right)^{2}} & \frac{\left[f_{a}^{2}\left(g_{1}^{2}+g_{2}^{2}\right)-4 \mu_{\text {eff }}^{2} t_{\beta}\left(t_{\beta}^{2}-1\right) v^{2}\right.}{2 f_{a}^{2}\left(t_{\beta}^{2}+1\right)^{2}} & \frac{2\left[\mu_{\text {eff }}^{2}\left(t_{\beta}^{2}+1\right)-B_{\text {eff }} t_{\beta}\right] v}{f_{a}\left(t_{\beta}^{2}+1\right)} \\
\frac{\left[f_{a}^{2}\left(g_{1}^{2}+g_{2}^{2}\right)-4 \mu_{\text {eff }}^{2}\right] t_{\beta}\left(t_{\beta}^{2}-1\right) v^{2}}{2 f_{a}^{2}\left(t_{\beta}^{2}+1\right)^{2}} & \frac{\left[f_{a}^{2}\left(g_{1}^{2}+g_{2}^{2}\right)-4 \mu_{\text {eff }}^{2}\right] v^{2} t_{\beta}^{3}+B_{\text {eff }} f_{a}^{2}\left(t_{\beta}^{2}+1\right)^{3}}{f_{a}^{2} t_{\beta}\left(t_{\beta}^{2}+1\right)^{2}} & \frac{B_{\text {eff }}\left(t_{\beta}^{2}-1\right) v}{f_{a}\left(t_{\beta}^{2}+1\right)} \\
\frac{2\left[\mu_{\text {eff }}^{2}\left(t_{\beta}^{2}+1\right)-B_{\text {eff }} t_{\beta}\right] v}{f_{a}\left(t_{\beta}^{2}+1\right)} & \frac{B_{\text {eff }}\left(t_{\beta}^{2}-1\right) v}{f_{a}\left(t_{\beta}^{2}+1\right)} & \frac{B_{\text {eff }} t_{\beta} v^{2}}{f_{a}^{2}\left(t_{\beta}^{2}+1\right)}
\end{array}\right) .
$$

Neglecting the entries with a $v^{2} / f_{a}^{2}$ suppression and approximating $t_{\beta}^{2}+1=t_{\beta}^{2}-1=t_{\beta}^{2}$ this matrix has the form

$$
\left(\begin{array}{ccc}
\frac{1}{4}\left(g_{1}^{2}+g_{2}^{2}\right) v^{2} & \frac{\left(g_{1}^{2}+g_{2}^{2}\right) v^{2}}{2 t_{\beta}} & \frac{2\left(\mu_{\text {eff }}^{2} t_{\beta}-B_{\text {eff }}\right) v}{f_{a} t_{\beta}} \\
\frac{\left(g_{1}^{2}+g_{2}^{2}\right) v^{2}}{2 t_{\beta}} & \frac{\left(g_{1}^{2}+g_{2}^{2}\right) v^{2}}{t_{\beta}^{2}}+B_{\text {eff }} t_{\beta} & \frac{B_{\text {eff }} v}{f_{a}} \\
\frac{2\left(\mu_{\text {eff }}^{2} t_{\beta}-B_{\text {eff }}\right) v}{f_{a} t_{\beta}} & \frac{B_{\text {eff }} v}{f_{a}} & 0
\end{array}\right) .
$$

The determinant is given by

$$
\begin{aligned}
-\frac{1}{4 f_{a}^{2} t_{\beta}^{4}}\left\{v^{4}\left(g_{1}^{2}+g_{2}^{2}\right)\left[B_{\mathrm{eff}}\left(t_{\beta}^{2}+4\right)-4 \mu_{\mathrm{eff}}^{2} t_{\beta}\right]^{2}\right. \\
\left.+16 B_{\mathrm{eff}} t_{\beta}^{3} v^{2}\left(B_{\mathrm{eff}}-\mu_{\mathrm{eff}}^{2} t_{\beta}\right)^{2}\right\} .
\end{aligned}
$$

$B_{\text {eff }}$ must be positive, otherwise the mass of the charged Higgs is below the $W$ boson mass. Hence the determinant is always negative and the saxion is a tachyon. This is a new result to the best of our knowledge. We conclude that this model is not consistent. As the issue can be traced back to the minimization condition, Eq. (9), corresponding to the PQ-breaking VEV, the problem can be fixed by adding terms in the superpotential to spontaneously break the PQ symmetry and stabilize the PQ breaking scale.

\section{A SELF-CONSISTENT MODEL}

We add the following terms [44] to the superpotential in Eq. (2)

$$
W_{\mathrm{PQ}}=\lambda \hat{\chi}\left(\hat{A} \hat{\bar{A}}-\frac{1}{4} f_{a}^{2}\right)
$$

with the distinct superfields $\hat{A}, \hat{\bar{A}}$, as well as $\hat{\chi}$. $\hat{\bar{A}}$ carries a PQ charge opposite to $\hat{A}$, while $\hat{\chi}$ is $\mathrm{PQ}$ neutral. Assuming that a global R symmetry [55] forbids terms quadratic and cubic in $\hat{\chi}$, we have written all the terms consistent with the gauge and PQ symmetries, as well as with R-parity.

Considering the superpotential $W_{\mathrm{PQ}}$ alone with unbroken SUSY, one has two heavy superfields, $\hat{\chi}$ and $\frac{1}{\sqrt{2}}(\hat{A}+\hat{\bar{A}})$, with masses $\sim f_{a}$, and a massless superfield,
$\frac{1}{\sqrt{2}}(\hat{A}-\hat{\bar{A}})$. The latter can be identified as the axion superfield. If SUSY is broken at a scale $M_{\mathrm{SB}} \ll f_{a}$ we can integrate out the heavy superfield in a supersymmetric fashion and then consider the SUSY breaking effects in the resulting effective theory. We will study this case in the next section. When $M_{\mathrm{SB}} \gg f_{a}$, like in supergravity models, we have to take into account the SUSY breaking effects also for the fields with masses $\sim f_{a}$. This is the case we consider in the rest of this section.

After electroweak symmetry breaking (EWSB) $\chi$ gets a VEV, $v_{\chi}$, thus the R symmetry is broken. The corresponding R-axion has a mass of order $M_{\mathrm{SUSY}}$ because the R-symmetry is also explicitly broken by the soft terms. Beyond those in Eq. (6) we have the soft-breaking terms

$$
V_{\mathrm{soft}}^{\mathrm{PQ}}=T_{\lambda} \chi A \bar{A}-L_{V} \chi+m_{\bar{a}}^{2}|\bar{A}|^{2}+m_{\chi}^{2}|\chi|^{2} .
$$

The trilinear and linear terms, with coefficients $T_{\lambda}$ and $L_{V}$, will play an important role when we discuss the mass of the axino. Note that one expects $L_{V} \sim M_{\mathrm{SUSY}} f_{a}^{2}$.

After PQ and EW breaking the fields $H_{d}, H_{u}, A, \bar{A}$ and $\chi$ receive $\mathrm{VEVs}$ :

$$
\begin{gathered}
A=\frac{1}{\sqrt{2}}\left(\phi_{a}+i \sigma_{a}+v_{a}\right), \quad \bar{A}=\frac{1}{\sqrt{2}}\left(\phi_{\bar{a}}+i \sigma_{\bar{a}}+v_{\bar{a}}\right) \\
\chi=\frac{1}{\sqrt{2}}\left(\phi_{\chi}+i \sigma_{\chi}+v_{\chi}\right),
\end{gathered}
$$

with $v_{a} v_{\bar{a}}=\frac{1}{2} f_{a}^{2}$. The fields $H_{u}, H_{d}$ are parametrized as in Eq. (10). The tadpole equations for $\phi_{a}, \phi_{\bar{a}}$ and $\chi$ read

$$
\begin{aligned}
\frac{\partial V}{\partial \phi_{a}} & =m_{a}^{2} v_{a}+\frac{1}{4}\left[2 v_{a}\left(c_{1}^{2}\left(v_{d}^{2}+v_{u}^{2}\right)\right)\right. \\
& \left.+2 \sqrt{2}\left(v_{\chi} v_{\bar{a}} T_{\lambda}-v_{d} v_{u} T_{c 1}\right)+2 \lambda^{2} v_{a} v_{\chi}^{2}\right]=0 \\
\frac{\partial V}{\partial \phi_{\bar{a}}} & =m_{\bar{a}}^{2} v_{\bar{a}}+\frac{1}{4}\left(-2 \lambda c_{1} v_{\chi} v_{d} v_{u}+2 \lambda^{2} v_{\bar{a}} v_{\chi}^{2}\right. \\
& \left.+2 \sqrt{2} v_{\chi} v_{a} T_{\lambda}\right)=0, \\
\frac{\partial V}{\partial \phi_{\chi}} & =m_{\chi}^{2} v_{\chi}-\sqrt{2} L_{V}+\frac{1}{2}\left[-\lambda c_{1} v_{d} v_{u} v_{\bar{a}}+\sqrt{2} v_{a} v_{\bar{a}} T_{\lambda}\right. \\
& \left.+v_{\chi} \lambda^{2}\left(v_{a}^{2}+v_{\bar{a}}^{2}\right)\right]=0 .
\end{aligned}
$$

We can consistently solve these equations keeping all the soft parameters at the $M_{\text {SUSY }}$ scale. In particular we can 
solve the last equation for $v_{\chi}$ and find

$v_{\chi}=\frac{2 \sqrt{2}}{\lambda^{2}\left(v_{a}^{2}+v_{\bar{a}}^{2}\right)} L_{V}-\frac{\sqrt{2} v_{a} v_{\bar{a}}}{\lambda^{2}\left(v_{a}^{2}+v_{\bar{a}}^{2}\right)} T_{\lambda}+\mathcal{O}\left(\frac{M_{\mathrm{SUSY}}^{2}}{f_{a}}\right)$.

This result will be important for the discussion below on the axino mass.

First we comment on the scalar masses in our model. Eq. (1) introduces a mixing between the MSSM Higgs sector and the axion sector. It turns out that the correction to the light Higgs mass, $m_{h}$, is of order $\mu_{\mathrm{eff}}^{2} / f_{a}$, which is negligible. As a consequence the usual upper limit $m_{h}<m_{Z}$ holds at tree level. On the other hand the tree-level mass of the heavy Higgs, $m_{H}$, is modified as

$$
m_{H}^{2}=\frac{\left(2 B_{\mathrm{eff}}+\sqrt{2} \mu_{\mathrm{eff}} v_{\chi} \lambda\right)}{\sin (2 \beta)}+\frac{v^{2}}{4} \sin ^{2}(2 \beta)\left(g_{1}^{2}+g_{2}^{2}\right) .
$$

Due to $v_{\chi} \neq 0$ this can be potentially different from the MSSM. If we neglect the small mixing between the MSSM and the axion sector the three squared mass eigenvalues stemming from the mixing among $\left(\phi_{a}, \phi_{\bar{a}}, \phi_{\chi}\right)$ are given by

$$
\frac{4 L_{V} T_{\lambda}+T_{\lambda}^{2} f_{a}^{2}}{f_{a}^{2} \lambda^{2}}, \frac{f_{a}^{3} \lambda^{2} \pm 4 \sqrt{2} L_{V}}{2 f_{a}} .
$$

The first is the smaller one and is associated it with the saxion mass squared. In models where the SUSY breaking effects are mediated by gravity, one expects the linear and trilinear soft terms to be of order $M_{\mathrm{SUSY}} \sim m_{3 / 2}$, with $m_{3 / 2}$ the gravitino mass. In such a scenario the saxion mass is then of order $M_{\mathrm{SUSY}}$. The other two scalars have a mass of order $f_{a}$. In the scalar CP-odd sector we find a massless axion [56] as expected.

The extended neutralino mass matrix reads, in the ba$\operatorname{sis}\left(\lambda_{\tilde{B}}, \tilde{W}^{0}, \tilde{H}_{u}^{0}, \tilde{H}_{d}^{0}, \tilde{A}, \tilde{\bar{A}}, \tilde{\chi}\right)$

$$
m_{\chi^{0}}=\left(\begin{array}{ccccccc}
M_{1} & 0 & \frac{1}{2} g_{1} v_{u} & -\frac{1}{2} g_{1} v_{d} & 0 & 0 & 0 \\
0 & M_{2} & -\frac{1}{2} g_{2} v_{u} & \frac{1}{2} g_{2} v_{d} & 0 & 0 & 0 \\
\frac{1}{2} g_{1} v_{u} & -\frac{1}{2} g_{2} v_{u} & 0 & -\frac{1}{\sqrt{2}} c_{1} v_{a} & -\frac{1}{\sqrt{2}} c_{1} v_{d} & 0 & 0 \\
-\frac{1}{2} g_{1} v_{d} & \frac{1}{2} g_{2} v_{d} & -\frac{1}{\sqrt{2}} c_{1} v_{a} & 0 & -\frac{1}{\sqrt{2}} c_{1} v_{u} & 0 & 0 \\
0 & 0 & -\frac{1}{\sqrt{2}} c_{1} v_{d} & -\frac{1}{\sqrt{2}} c_{1} v_{u} & 0 & \frac{1}{\sqrt{2}} v_{\chi} \lambda & \frac{1}{\sqrt{2}} v_{\bar{a}} \lambda \\
0 & 0 & 0 & 0 & \frac{1}{\sqrt{2}} v_{\chi} \lambda & 0 & \frac{1}{\sqrt{2}} v_{a} \lambda \\
0 & 0 & 0 & 0 & \frac{1}{\sqrt{2}} v_{\bar{a}} \lambda & \frac{1}{\sqrt{2}} v_{a} \lambda & 0
\end{array}\right) .
$$

In the limit $v_{a}=v_{\bar{a}}=\frac{f_{a}}{\sqrt{2}}, c_{1} v_{u} \rightarrow 0, c_{1} v_{d} \rightarrow 0$ the lower right $3 \times 3$ block has the singular values [45]

$$
-\frac{1}{\sqrt{2}} v_{\chi} \lambda, \quad \frac{1}{2 \sqrt{2}}\left( \pm \sqrt{v_{\chi}^{2}+4 f_{a}^{2}} \lambda+v_{\chi} \lambda\right) .
$$

The first is associated with the phyiscal axino. Its mass is proportional to $v_{\chi}$, therefore of order $M_{\mathrm{SUSY}}$. It is important to notice that the fact that the field $\chi$ develops a VEV $v_{\chi}$ is tightly connected to the SUSY breaking effects. If the SUSY breaking scale is much lower than the PQ scale, we can integrate out the heavy superfield $\hat{\chi}$ supersymmetrically, after which there would be no notion of $v_{\chi}$ any longer. When we study this limit in the next section we will see that the resulting axino is very light.

In the model considered in this section we also have an extra handle on the axino mass. We can relax the assumption $v_{a}=v_{\bar{a}}=\frac{f_{a}}{\sqrt{2}}$ and consider a hierarchy between the two VEVs, for example $v_{\bar{a}} \gg v_{a}$. If we do so we find that the axino mass becomes lighter. In the limit $v_{a} \rightarrow 0$, keeping fixed $v_{a} v_{\bar{a}}=1 / 2 f_{a}^{2}$, the axino mass tends to zero. We show the axino mass as a function of $\tan \beta^{\prime}=\sqrt{v_{\bar{a}} / v_{a}}$ in Fig. 1 .

\section{Renormalization Group Equations}

The full model defined by the superpotential terms in Eqs. (2) and (16) can be defined as the minimal supersymmetric DFSZ axion model. It is possible at this point to make some simplifying assumptions in a CMSSM fashion, and study phenomenology and constraints with a relatively small parameter space. This will be studied in a future work in detail but here we briefly comment on the main aspects.

In the Appendix we give the one-loop RGEs for the axino sector and the changes in the $\beta$ functions. As already stated in the introduction, the $\beta$ functions of the MSSM parameters change only by terms of order $\mu / f_{a}$, which is usually a negligible effect. This is in contrast to the NMSSM, where the coupling between the Higgs doublets and the gauge singlet, the analogous of our $c_{1}$, can be of order one and can significantly change the MSSM RGEs.

In the CMSSM one uses universal boundary conditions. In the same spirit, the simplest choice to keep the number of parameters to a minimum is to use the same boundary conditions also for the parameters in our new sector. Thus, our additional soft terms at the GUT 


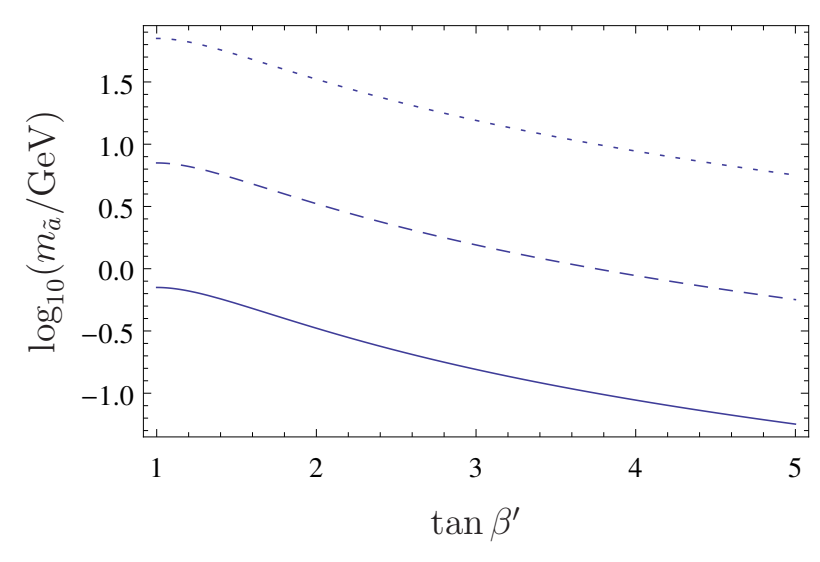

FIG. 1: The mass of the axino as a function of $\tan \beta^{\prime}=$ $\sqrt{v_{\bar{a}} / v_{a}}$. We fixed $f_{a}=10^{12} \mathrm{GeV}, \lambda=10^{-2}, T_{\lambda}=-1 \mathrm{TeV}$ and used $v_{\chi}=10^{4} \mathrm{GeV}$ (dotted line), $v_{\chi}=10^{3} \mathrm{GeV}$ (dashed line), $v_{\chi}=10^{2} \mathrm{GeV}$ (plain line).

scale are fixed by two parameters, $m_{0}^{2}$ and $A_{0}$ :

$$
\begin{gathered}
m_{a}^{2}=m_{\bar{a}}^{2}=m_{\chi}^{2} \equiv m_{0}^{2}, \\
T_{\lambda} \equiv A_{0} \lambda, \quad T_{c 1} \equiv A_{0} c_{1}
\end{gathered}
$$

while $L_{V}$ can be eliminated by using the minimization conditions of the vacuum. With this setup, we have immediately a lower limit on $m_{0}$ of several hundreds of $\mathrm{GeV}$ coming from squark searches at the LHC. If we neglect the small contributions from $c_{1}$ and $T_{c_{1}}$, the relation $m_{a}^{2}=m_{\tilde{a}}^{2}=m_{\chi^{2}} \equiv m^{2}$ holds at each scale and we have effectively the three RGEs

$$
\begin{aligned}
16 \pi^{2} \frac{d}{d t} m^{2} & =6 m^{2}|\lambda|^{2}+2\left|T_{\lambda}\right|^{2}, \\
16 \pi^{2} \frac{d}{d t} \lambda & =3 \lambda|\lambda|^{2}, \\
16 \pi^{2} \frac{d}{d t} T_{\lambda} & =9 T_{\lambda}|\lambda|^{2},
\end{aligned}
$$

which can even be solved analytically:

$$
\begin{aligned}
\lambda(t) & =\frac{2 \pi}{\sqrt{\frac{3\left(t_{G U T}-t\right)}{2}+\frac{4 \pi^{2}}{\lambda^{2}}}}, \\
T_{\lambda}(t) & =-\frac{16 i \sqrt{2} \pi^{3} A_{0}}{\lambda^{2}\left(3\left(t-t_{G U T}\right)-\frac{8 \pi^{2}}{\lambda^{2}}\right)^{3 / 2}}, \\
m^{2}(t) & =\frac{64 \pi^{4} m_{0}^{2}-8 \pi^{2} \lambda^{2}\left(A_{0}^{2}-3 m_{0}^{2}\right)\left(t_{G U T}-t\right)}{\left(3 \lambda^{2}\left(t_{G U T}-t\right)+8 \pi^{2}\right)^{2}} .
\end{aligned}
$$

Here $t$ is the renormalization scale and $t_{G U T}$ the scale of grand unification where the boundary conditions have been applied.

\section{THE AXINO MASS}

In the above discussion we have parametrized the SUSY breaking effects in the soft terms and assumed a high SUSY breaking scale, $M_{\mathrm{SB}} \gg f_{a}$. We have also seen that we have some heavy fields in the spectrum, with masses of order $f_{a}$. In this section we study how integrating out the heavy fields affects the mass of the axino in the low energy theory. We distinguish two cases. In the first we consider $M_{\mathrm{SB}} \gg f_{a}$, as in the previous section. Here we find that the axino mass remains of order $M_{\mathrm{SUSY}}$. In the second we take the opposite limit, $M_{\mathrm{SB}} \ll f_{a}$, and find that the resulting axino is much lighter.

\section{High scale SUSY breaking}

We have seen in Eq. 26) that retaining all the fields we obtain an axino mass of order $M_{\mathrm{SUSY}}$. One may wonder what happens to the axino mass in the low-energy theory if we integrate out the heavy fields in this scenario. We have to integrate them out component by component as SUSY is already broken. First we diagonalize the scalar and fermionic mass matrices. As we have seen in eq. (24), it is easy to identify in the CP-even sector the light state, the saxion, which we denote $\phi_{a}^{\text {even }}$ here, and the two heavy scalar states, with masses of order $f_{a}$, which we denote $\phi_{b}^{\text {even }}$ and $\phi_{c}^{\text {even }}$. In the fermionic sector we have the axino, $\psi_{a}$, associated with the first eigenvalue in eq. (26), and two heavy fermions, $\psi_{b}$ and $\psi_{c}$, associated with the other two eigenvalues. At tree level the only contributions to the axino mass one can have when integrating out the heavy fields is depicted in Fig. 2, Note that only the CP-even scalars contribute. It is easy to check that the yukawa coupling between the scalar and the two $\psi_{a}$ 's is the same for $\phi_{b}^{\text {even }}$ and $\phi_{c}^{\text {even }}$. The scalar propagator ends in a tadpole. This is the key point. The tadpole is given by $\frac{\partial V}{\partial \phi_{i}^{\text {even }}}$, with $V$ the scalar potential. Then one is guaranteed that the tadpoles for $\phi_{b}^{\text {even }}$ and $\phi_{c}^{\text {even }}$ are zero, as this corresponds to the minimization condition of the scalar potential. In the previous section we used $\frac{\partial V}{\partial \phi_{i}}=0$ for the gauge eigenstates, $\phi_{i}$. It is clear that the same condition holds for the mass eigenstates $\phi_{i}^{\text {even }}$ here, as we have $\frac{\partial V}{\partial \phi_{i}^{\text {even }}}=\frac{\partial \phi_{j}}{\partial \phi_{i}^{\text {even }}} \frac{\partial V}{\partial \phi_{j}}=0$. Thus the contribution of the diagrams in Fig. 2 vanishes and the axino mass at tree level does not change in the low energy. One might worry about loop corrections. At worst these would be of order $\frac{1}{16 \pi^{2}} M_{\mathrm{SUSY}}$. Because of the $16 \pi^{2}$ loop-suppression they would not provide any significant cancelation. We conclude that the axino mass in this scenario remains of order $M_{\mathrm{SUSY}}$. 


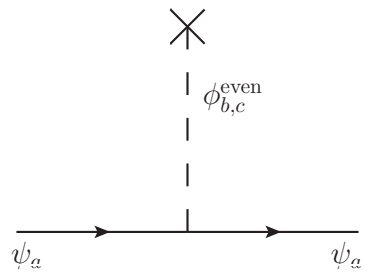

FIG. 2: Diagrams contributing to the axino mass when integrating out the heavy fields $\phi_{b, c}$.

\section{Low scale SUSY breaking}

If $M_{\mathrm{SB}} \ll f_{a}$ SUSY is still unbroken at the PQ scale and we can perform the following redefinitions of the superfields:

$$
\begin{aligned}
& \hat{\chi} \rightarrow \hat{\chi} \\
& \hat{A} \rightarrow\left(\frac{1}{2} f_{a}+\frac{1}{\sqrt{2}} \hat{\Phi}_{H}\right) e^{\sqrt{2} \frac{\hat{\Phi}_{a}}{f_{a}}}, \\
& \hat{\bar{A}} \rightarrow\left(\frac{1}{2} f_{a}+\frac{1}{\sqrt{2}} \hat{\Phi}_{H}\right) e^{-\sqrt{2} \frac{\hat{\Phi}_{a}}{f_{a}}} .
\end{aligned}
$$

Inserting into Eq. (16) we see that the superfields $\hat{\chi}$ and $\hat{\Phi}_{H}$ have masses of order $f_{a}$, while $\hat{\Phi}_{a}$ is massless. The latter is the axion superfield. This parametrization [21] is useful because it explicitly shows that the original PQ transformation $\hat{A} \rightarrow e^{i \alpha} \hat{A}$ is now encoded in $\hat{\Phi}_{a} \rightarrow \hat{\Phi}_{a}+i \sqrt{2} \alpha f_{a}$. We recognize here the shift symmetry typical of axions that must be respected in the low energy theory. Let's consider the superpotential in terms of the new superfields

$$
\begin{aligned}
W_{2}= & \frac{\lambda}{2} \hat{\chi} \hat{\Phi}_{H}\left(\hat{\Phi}_{H}+\sqrt{2} f_{a}\right) \\
& +c_{1}\left(\frac{1}{2} f_{a}+\frac{1}{\sqrt{2}} \hat{\Phi}_{H}\right) e^{\sqrt{2} \frac{\hat{\Phi}_{a}}{f_{a}}} \hat{H}_{u} \hat{H}_{d} .
\end{aligned}
$$

We can integrate out the heavy fields in a supersymmetric fashion using their equations of motion: $\frac{\partial W_{2}}{\partial \hat{\chi}}=0$ and $\frac{\partial W_{2}}{\partial \hat{\Phi}_{H}}=0$. We find the following effective superpotential

$W_{\text {eff }}=\mu_{\text {eff }} \hat{H}_{u} \hat{H}_{d}+\frac{c_{1}}{\sqrt{2}} \hat{\Phi}_{a} \hat{H}_{u} \hat{H}_{d}+\frac{c_{1}}{2} \sum_{n \geq 2} \frac{\left(\sqrt{2} \hat{\Phi}_{a}\right)^{n}}{f_{a}^{n-1}} \hat{H}_{u} \hat{H}_{d}$

In the first term we have $\mu_{\text {eff }}=\frac{c_{1}}{2} f_{a}$, while the last term contains higher dimension operators that we can safely neglect because they are suppressed by increasing negative powers of $f_{a}$. Next we consider the effects of SUSY breaking. The soft terms for the low energy field content read

$$
\begin{aligned}
V_{\mathrm{soft}}= & T_{u} \tilde{u} \tilde{q} H_{u}+T_{d} \tilde{d} \tilde{q} H_{d}+T_{e} \tilde{e} \tilde{l} H_{d}+T_{c 1} \Phi_{a} H_{u} H_{d} \\
& +B_{\mathrm{eff}} H_{u} H_{d}+m_{H_{u}}^{2}\left|H_{u}\right|^{2}+m_{H_{d}}^{2}\left|H_{d}\right|^{2}+\tilde{\phi}^{\dagger} m_{\tilde{\phi}}^{2} \tilde{\phi} \\
& +m_{a}^{2}\left(\Phi_{a}+\Phi_{a}^{*}\right)^{2} .
\end{aligned}
$$

The form of the last term is dictated by the shift symmetry, which would be otherwise violated if we wrote $m_{a}^{2}\left|\Phi_{a}\right|^{2}$. Indeed this term in Eq. (38) gives a mass to the saxion, the real part of $\Phi_{a}$, but leaves the axion massless. Note that after integrating out the heavy fields, the parameters $\lambda, T_{\lambda}, L_{V}$ do not appear any more in the low energy lagrangian. We parametrize the fields $H_{u}$ and $H_{d}$ as in Eq. 10, but do not assign a VEV to the field $\Phi_{a}$, as that would break the shift symmetry. Thus we write $\Phi_{a}=\frac{1}{\sqrt{2}}\left(\phi_{a}+i \sigma_{a}\right)$. The tadpole equations read

$$
\begin{aligned}
\left.\frac{\partial V}{\partial \phi_{d}}\right|_{\phi=\sigma=0}= & m_{H_{d}}^{2} v_{d}+\frac{1}{8}\left[2 c_{1}^{2} v_{d} v_{u}^{2}+8 \mu_{\mathrm{eff}}^{2} v_{d}-8 v_{u} B_{\mathrm{eff}}\right. \\
& \left.+v_{d}\left(g_{1}^{2}+g_{2}^{2}\right)\left(v_{d}^{2}-v_{u}^{2}\right)\right]=0 \\
\left.\frac{\partial V}{\partial \phi_{u}}\right|_{\phi=\sigma=0}= & m_{H_{u}}^{2} v_{u}+\frac{1}{8}\left[2 c_{1}^{2} v_{u} v_{d}^{2}+8 \mu_{\mathrm{eff}}^{2} v_{u}-8 v_{d} B_{\mathrm{eff}}\right. \\
& \left.-v_{u}\left(g_{1}^{2}+g_{2}^{2}\right)\left(v_{d}^{2}-v_{u}^{2}\right)\right]=0 \\
\left.\frac{\partial V}{\partial \phi_{a}}\right|_{\phi=\sigma=0}= & v_{d} v_{u} T_{c 1}-c_{1} \mu_{\mathrm{eff}}\left(v_{d}^{2}+v_{u}^{2}\right)=0
\end{aligned}
$$

We see at this stage that the issue which made the model of the first section inconsistent is no longer present. The parameter $m_{a}$ is absent from these equations. Thus we can retain all the soft masses at the $M_{\text {SUSY }}$ scale. We find that the Higgs masses are now the same as in the MSSM, up to tiny corrections proportional to the small parameter $c_{1}$. The correction to the heavy Higgs mass in Eq. 23 proportional to $v_{\chi}$ is no longer present, as $v_{\chi}$ is effectively zero in the limit $M_{\mathrm{SB}} \ll f_{a}$. The axion is massless.

The saxion mass comes almost entirely from the soft parameter $m_{a}$, as the contributions from the mixing with the Higgs fields are suppressed by the tiny value of $c_{1}$, and it deserves a comment. If one considered minimal gauge mediation as an example of low scale SUSY breaking, then the parameter $m_{a}$ would only be generated at three loops, as $\Phi_{a}$ is a gauge singlet. The saxion would then be very light and very problematic from the cosmological point of view 46. One could easily contemplate extended hidden sectors in the context of gauge mediation which would result in a heavier saxion [47. Such extensions would likely produce a mass for the axino comparable to that of the saxion. However, it is our aim here to keep our model minimal in order to make it as predictive as possible. Thus we do not consider any hidden sector or mediation mechanism but just parametrize the saxion mass as $m_{a}$. This is a free parameter for us, which can be taken to be of order $M_{\mathrm{SUSY}}$, to avoid cosmological problems. A further clarification is then necessary. In Ref. 32 the authors claimed that in theories with spontaneously broken SUSY with $M_{\mathrm{SB}} \ll f_{a}$ the saxion mass is at most $M_{\mathrm{SUSY}}^{2} / f_{a}$. Their result relies on the assumption that the supertrace sum rule [48] holds. The inclusion of the explicit soft SUSY breaking terms vio- 
lates this assumption, and our saxion mass comes indeed from the soft term. Therefore our result is not in conflict with Ref. 32.

Let us consider the fermions. In the basis
$\left(\lambda_{\tilde{B}}, \tilde{W}^{0}, \tilde{H}_{u}^{0}, \tilde{H}_{d}^{0}, \tilde{\Phi}_{a}\right)$ the $5 \times 5$ extended neutralino mass matrix reads

$$
m_{\chi^{0}}=\left(\begin{array}{ccccc}
M_{1} & 0 & \frac{1}{2} g_{1} v_{u} & -\frac{1}{2} g_{1} v_{d} & 0 \\
0 & M_{2} & -\frac{1}{2} g_{2} v_{u} & \frac{1}{2} g_{2} v_{d} & 0 \\
\frac{1}{2} g_{1} v_{u} & -\frac{1}{2} g_{2} v_{u} & 0 & -\mu_{\mathrm{eff}} & -\frac{1}{2} c_{1} v_{d} \\
-\frac{1}{2} g_{1} v_{d} & \frac{1}{2} g_{2} v_{d} & -\mu_{\mathrm{eff}} & 0 & -\frac{1}{2} c_{1} v_{u} \\
0 & 0 & -\frac{1}{2} c_{1} v_{d} & -\frac{1}{2} c_{1} v_{u} & 0
\end{array}\right) .
$$

The smallest eigenvalue here is of order $c_{1} v$, with $v$ of order the EWSB VEV, and it corresponds to the axino. Given that $c_{1}=\frac{\mu_{\text {eff }}}{f_{a}}$, the axino in this model has a mass of order $M_{\text {SUSY }}^{2} / f_{a} \leq \mathcal{O}(\mathrm{keV})$.

\section{Comments}

The axino mass has been widely discussed in the literature. Tamvakis and Wyler 32 showed that in models with global SUSY the axino mass would be at most of order $\mathcal{O}\left(\frac{M_{\mathrm{SUSY}}^{2}}{f_{a}}\right)$ after SUSY breaking. Chun, Kim, Lukas and Nilles [33, 34 found that in models with local SUSY, i.e. supergravity, the axino mass can have a wider range and can be as large as the gravitino mass, $m_{3 / 2}$. Our results agree with those statements. Indeed a low SUSY breaking scale, for which we find a light axino, is typical of models with global SUSY, while a higher scale, $M_{\mathrm{SB}} \geq f_{a}$, for which we find a heavier axino, is representative of supergravity. In the latter case we can identify the scale of our soft terms with the gravitino mass, $M_{\text {SUSY }} \sim m_{3 / 2} \sim \frac{F}{M_{p}}$, with $M_{p}$ the Planck mass.

We emphasize, however, that the distinction between models of global SUSY breaking and supergravity is not strictly related to the scale $M_{\mathrm{SB}}$. Recently, for example, gauge mediation models with a high scale, $M_{\mathrm{SB}}>f_{a}$, have been considered (see e.g. [49]). Our statements on the axino mass only refer to the relative size of the scales $M_{\mathrm{SB}}$ and $f_{a}$ and make no explicit reference to the SUSY breaking mechanism.

Our results apply as long as the scales $f_{a}$ and $M_{\mathrm{SB}}$ are well separated. The case $f_{a} \simeq M_{S B}$ would require a special treatment because it is no longer valid to consider the SUSY breaking effects to appear/disappear instantaneously. However this is not possible in a momentum independent renormalization scheme like $\overline{\mathrm{DR}}$ which treats thresholds as step functions [50], and is beyond the scope of this discussion here.

\section{AXION/AXINO COUPLINGS TO GAUGE FIELDS}

The most important feature of an axion model is that it must solve the strong $\mathrm{CP}$ problem. This is achieved thanks to the fact that the $U(1)_{\mathrm{PQ}}$ is anomalous, which generates the following coupling of the axion to the gluons

$$
\mathcal{L}_{a g g}=\frac{\alpha_{s}}{8 \pi} \frac{a_{\mathrm{phys}}}{f_{a}} G_{\mu \nu}^{a} \tilde{G}^{a \mu \nu}
$$

Here $\alpha_{s}=\frac{g_{s}^{2}}{4 \pi}$, with $g_{s}$ the strong coupling constant, $a_{\text {phys }}$ is the axion field, i.e. the massless eigenstate in the scalar CP-odd sector, $G_{\mu \nu}^{a}$ the gluon field strength, and $\tilde{G}^{a \mu \nu} \equiv \epsilon^{\mu \nu \rho \sigma} G_{\rho \sigma}^{a}$. This anomalous coupling in our model is exactly the same as in the original non-SUSY version of the DFSZ model [13], and generates a small axion mass, $m_{a}$, such that $m_{a} f_{a} \simeq m_{\pi} f_{\pi}$, where $m_{\pi}$ and $f_{\pi}$ are the pion mass and decay constant.

The SUSY model defined in the previous section departs from its non-SUSY counterpart [13] for what concerns the coupling of axions to photons, which we parametrize as 51]

$$
\mathcal{L}_{a \gamma \gamma}=\frac{G_{a \gamma \gamma}}{4} a_{\mathrm{phys}} F_{\mu \nu} \tilde{F}^{\mu \nu}
$$

where

$$
G_{a \gamma \gamma}=\frac{\alpha}{2 \pi f_{a}}\left(\frac{E}{N}-\frac{2}{3} \frac{4+z}{1+z}\right),
$$

with $\alpha$ the fine structure constant, $E$ and $N$ the electromagnetic and color anomalies of the $U(1)_{\mathrm{PQ}}$ current, $z \equiv m_{u} / m_{d}$. In the original DFSZ model [13] one has $E=\frac{4}{3} N_{g}\left(X_{H_{u}}+X_{H_{d}}\right)$ and $N=\frac{1}{2} N_{g}\left(X_{H_{u}}+X_{H_{d}}\right)$, which results in $E / N=8 / 3$. Here $X_{H_{u}}$ and $X_{H_{d}}$ are the PQ charges of the corresponding Higgs doublets, $N_{g}$ is the number of quark and lepton generations. In our SUSY model there is an extra contribution to the electromagnetic anomaly which comes from the electrically charged 
higgsinos. Including this contribution we find

$$
\begin{aligned}
E & =\left(\frac{4}{3} N_{g}+1\right)\left(X_{H_{u}}+X_{H_{d}}\right) \\
\frac{E}{N} & =\frac{2}{N_{g}}\left(\frac{4}{3} N_{g}+1\right)=\frac{10}{3}
\end{aligned}
$$

where in the last equality we have set $N_{g}=3$. Thus the coupling to photons, which is crucial to many experimental axion searches, is slightly modified compared to the original model [13].

The axino couplings to gauge fields can also be relevant, in particular to study the thermal production of the axino in cosmology (see e.g. Refs. [19, 20, 52]). The form of the operators for the interactions axino - gaugino - gauge boson can be obtained via supersymmetrization of eq. (43) and eq. 44. However the coefficients of these operators will be different as the physical axino is a slightly different linear combination of the fields $\hat{A}, \hat{\bar{A}}, \hat{\chi}, \hat{H}_{u}, \hat{H}_{d}$, compared to the axion. Such coefficients can be calculated numerically in our model, but this is beyond the scope of the current work.

\section{CONCLUSION}

We have investigated what are the minimal ingredients needed to define a consistent minimal supersymmetric DFSZ axion model. We have pointed out that the simplest model, which was first proposed in Ref. [15], is inconsistent as it suffers from a tachyonic saxion. The issue is solved by extending the superpotential to stabilize the PQ scale. We have then considered two cases: one where the SUSY breaking scale is much lower than the PQ breaking scale, the other where the two scales are comparable. In both cases the axion remains massless, as it should, and the saxion gets a mass of order $M_{\mathrm{SUSY}}$ (or $m_{3 / 2}$ ), roughly in the $\mathrm{TeV}$ range. The axino mass is dramatically different depending on the scenario. In the first $\left(M_{\mathrm{SB}} \ll f_{a}\right)$ it is very light, below the $\mathrm{keV}$ scale, while in the second $\left(M_{\mathrm{SB}} \geq f_{a}\right)$ it can be as large as the saxion mass. These results are in agreement with previous statements in the literature. Furthermore, in the second case, the mixing between the new states and the MSSM Higgs sector doesn't affect the mass of the light Higgs but can change the prediction for the heavy Higgs mass.

We have also discussed the couplings of the axion to gluons and photons. For the latter we found that the presence of charged higgsinos in the SUSY model slightly modifies the strength of the coupling, which could have implications for some experimental axion searches.

\section{Acknowledgements}

We thank Branislav Poletanović for stimulating this work, Hans-Peter Nilles for clarifying discussions, and Michael Dine for reading a preliminary version of this manuscript. HD and LU acknowledge the DFG SFB TR 33 'The Dark Universe' for support throughout this work.

\section{RGEs}

In this appendix we give the one-loop RGEs for the model of Section]. For each parameter $X$ they are defined by the following equation:

$$
\frac{d}{d t} X=\frac{1}{16 \pi^{2}} \beta_{X}^{(1)}
$$

with $t=\log Q$, where $Q$ is the renormalization scale. For parameters already present in the MSSM we show only the difference to the corresponding RGE in the MSSM

$$
\Delta \beta_{X}^{(1)}=\beta_{X}^{(1)}-\beta_{X}^{(1), M S S M} .
$$

The RGEs have been calculated using the generic expressions of Ref. 3] which are implemented in SARAH [40].

\section{Trilinear Superpotential Parameters}

$$
\begin{aligned}
\beta_{c_{1}}^{(1)} & =-3 c_{1} g_{2}^{2}+3 c_{1} \operatorname{Tr}\left(Y_{d} Y_{d}^{\dagger}\right)+3 c_{1} \operatorname{Tr}\left(Y_{u} Y_{u}^{\dagger}\right) \\
& +4 c_{1}^{2} c_{1}^{*}+c_{1}|\lambda|^{2}+c_{1} \operatorname{Tr}\left(Y_{e} Y_{e}^{\dagger}\right)-\frac{3}{5} c_{1} g_{1}^{2} \\
\beta_{\lambda}^{(1)} & =\lambda\left(2\left|c_{1}\right|^{2}+3|\lambda|^{2}\right) \\
\Delta \beta_{Y_{d}}^{(1)} & =Y_{d}\left|c_{1}\right|^{2} \\
\Delta \beta_{Y_{e}}^{(1)} & =Y_{e}\left|c_{1}\right|^{2} \\
\Delta \beta_{Y_{u}}^{(1)} & =Y_{u}\left|c_{1}\right|^{2}
\end{aligned}
$$

\section{Linear Superpotential Parameters}

$$
\beta_{f_{a}^{2}}^{(1)}=f_{a}^{2}|\lambda|^{2}
$$




$$
\begin{aligned}
\beta_{T_{c 1}}^{(1)} & =+T_{c 1}\left(12\left|c_{1}\right|^{2}-3 g_{2}^{2}+3 \operatorname{Tr}\left(Y_{d} Y_{d}^{\dagger}\right)+3 \operatorname{Tr}\left(Y_{u} Y_{u}^{\dagger}\right)\right. \\
& \left.-\frac{3}{5} g_{1}^{2}+|\lambda|^{2}+\operatorname{Tr}\left(Y_{e} Y_{e}^{\dagger}\right)\right) \\
& +\frac{2}{5} c_{1}\left(15 g_{2}^{2} M_{2}+15 \operatorname{Tr}\left(Y_{d}^{\dagger} T_{d}\right)+15 \operatorname{Tr}\left(Y_{u}^{\dagger} T_{u}\right)+\right. \\
& \left.3 g_{1}^{2} M_{1}+5 \lambda^{*} T_{\lambda}+5 \operatorname{Tr}\left(Y_{e}^{\dagger} T_{e}\right)\right) \\
\beta_{T_{\lambda}}^{(1)} & =2 c_{1}^{*}\left(2 \lambda T_{c 1}+c_{1} T_{\lambda}\right)+9|\lambda|^{2} T_{\lambda} \\
\Delta \beta_{T_{d}}^{(1)} & =\left|c_{1}\right|^{2} T_{d}+2 Y_{d} c_{1}^{*} T_{c 1} \\
\Delta \beta_{T_{e}}^{(1)} & =\left|c_{1}\right|^{2} T_{e}+2 Y_{e} c_{1}^{*} T_{c 1} \\
\Delta \beta_{T_{u}}^{(1)} & =\left|c_{1}\right|^{2} T_{u}+2 Y_{u} c_{1}^{*} T_{c 1}
\end{aligned}
$$

\section{Linear Soft-Breaking Parameters}

$$
\beta_{L_{V}}^{(1)}=\lambda^{*}\left(\frac{1}{2} f_{a}^{2} T_{\lambda}+\lambda L_{V}\right)
$$

\section{Soft-Breaking Scalar Masses}

$$
\begin{aligned}
\beta_{m_{a}^{2}}^{(1)} & =2\left(2\left(m_{a}^{2}+m_{H_{d}}^{2}+m_{H_{u}}^{2}\right)\left|c_{1}\right|^{2}+2\left|T_{c 1}\right|^{2}\right. \\
& \left.+\left(m_{a}^{2}+m_{\bar{a}}^{2}+m_{\chi}^{2}\right)|\lambda|^{2}+\left|T_{\lambda}\right|^{2}\right) \\
\beta_{m_{\bar{a}}^{2}}^{(1)} & =2\left(\left(m_{a}^{2}+m_{\bar{a}}^{2}+m_{\chi}^{2}\right)|\lambda|^{2}+\left|T_{\lambda}\right|^{2}\right) \\
\beta_{m_{\chi}^{2}}^{(1)} & =2\left(\left(m_{a}^{2}+m_{\bar{a}}^{2}+m_{\chi}^{2}\right)|\lambda|^{2}+\left|T_{\lambda}\right|^{2}\right) \\
\Delta \beta_{m_{H_{d}}^{2}}^{(1)} & =+2 m_{a}^{2}\left|c_{1}\right|^{2}+2\left|T_{c 1}\right|^{2} \\
\Delta \beta_{m_{H_{u}}^{2}}^{(1)} & =+2 m_{a}^{2}\left|c_{1}\right|^{2}+2\left|T_{c 1}\right|^{2}
\end{aligned}
$$

\section{Vacuum expectation values}

$$
\begin{aligned}
\beta_{v_{x}}^{(1)} & =-v_{x}\left(2\left|c_{1}\right|^{2}+|\lambda|^{2}\right) \\
\beta_{v_{\bar{x}}}^{(1)} & =-v_{\bar{x}}|\lambda|^{2} \\
\beta_{v_{\chi}}^{(1)} & =-v_{\chi}|\lambda|^{2} \\
\Delta \beta_{v_{d}}^{(1)} & =-v_{d}\left|c_{1}\right|^{2} \\
\Delta \beta_{v_{u}}^{(1)} & =-v_{u}\left|c_{1}\right|^{2}
\end{aligned}
$$

* Electronic address: dreiner@th.physik.uni-bonn.de

$\dagger$ Electronic address: fnstaub@th.physik.uni-bonn.de

¥ Electronic address: ubaldi@th.physik.uni-bonn.de

[1] S. P. Martin (1997), hep-ph/9709356.

[2] M. Drees and M. M. Nojiri, Phys.Rev. D47, 376 (1993), hep-ph/9207234.

[3] S. P. Martin and M. T. Vaughn, Phys.Rev. D50, 2282 (1994), hep-ph/9311340.

[4] I. Jack and D. Jones, Phys.Lett. B333, 372 (1994), hep$\mathrm{ph} / 9405233$.

[5] P. Bechtle, K. Desch, H. K. Dreiner, M. Hamer, M. KrŁmer, et al. (2013), 1310.3045.

[6] O. Buchmueller, R. Cavanaugh, A. De Roeck, M. Dolan, J. Ellis, et al., Eur.Phys.J. C72, 2020 (2012), 1112.3564.

[7] R. Peccei, Adv.Ser.Direct.High Energy Phys. 3, 503 (1989).

[8] S. Weinberg, Phys.Rev.Lett. 40, 223 (1978).

[9] F. Wilczek, Phys.Rev.Lett. 40, 279 (1978).

[10] J. E. Kim, Phys.Rev.Lett. 43, 103 (1979).

[11] M. A. Shifman, A. Vainshtein, and V. I. Zakharov, Nucl.Phys. B166, 493 (1980).

[12] R. Peccei and H. R. Quinn, Phys.Rev.Lett. 38, 1440 (1977).

[13] M. Dine, W. Fischler, and M. Srednicki, Phys.Lett. B104, 199 (1981).

[14] A. Zhitnitsky, Sov.J.Nucl.Phys. 31, 260 (1980).

[15] K. Rajagopal, M. S. Turner, and F. Wilczek, Nucl.Phys. B358, 447 (1991).

[16] L. Covi, H.-B. Kim, J. E. Kim, and L. Roszkowski, JHEP 0105, 033 (2001), hep-ph/0101009.

[17] H.-B. Kim and J. E. Kim, Phys.Lett. B527, 18 (2002), hep-ph/0108101.

[18] L. Covi and J. E. Kim, New J.Phys. 11, 105003 (2009), 0902.0769 .

[19] K.-Y. Choi, L. Covi, J. E. Kim, and L. Roszkowski, JHEP 1204, 106 (2012), 1108.2282.

[20] K. J. Bae, E. J. Chun, and S. H. Im, JCAP 1203, 013 (2012), 1111.5962.

[21] K. J. Bae, K. Choi, and S. H. Im, JHEP 1108, 065 (2011), 1106.2452.

[22] A. Strumia, JHEP 1006, 036 (2010), 1003.5847.

[23] H. Baer, M. Haider, S. Kraml, S. Sekmen, and H. Summy, JCAP 0902, 002 (2009), 0812.2693.

[24] H. Baer, A. D. Box, and H. Summy, JHEP 0908, 080 (2009), 0906.2595.

[25] H. Baer and A. D. Box, Eur.Phys.J. C68, 523 (2010), 0910.0333.

[26] H. Baer, A. D. Box, and H. Summy, JHEP 1010, 023 (2010), 1005.2215.

[27] H. Baer, A. Lessa, S. Rajagopalan, and W. Sreethawong, JCAP 1106, 031 (2011), 1103.5413.

[28] H. Baer and A. Lessa, JHEP 1106, 027 (2011), 1104.4807 .

[29] K. J. Bae, H. Baer, and E. J. Chun, JCAP 1312, 028 (2013), 1309.5365.

[30] H. Baer, V. Barger, P. Huang, A. Mustafayev, and X. Tata, Phys.Rev.Lett. 109, 161802 (2012), 1207.3343.

[31] F. E. Paige, S. D. Protopopescu, H. Baer, and X. Tata (2003), hep-ph/0312045.

[32] K. Tamvakis and D. Wyler, Phys.Lett. B112, 451 (1982).

[33] E. Chun, J. E. Kim, and H. P. Nilles, Phys.Lett. B287, 
123 (1992), hep-ph/9205229.

[34] E. Chun and A. Lukas, Phys.Lett. B357, 43 (1995), hep$\mathrm{ph} / 9503233$.

[35] J. E. Kim and H. P. Nilles, Phys.Lett. B138, 150 (1984).

[36] G. Honecker and W. Staessens, Fortsch.Phys. 62, 115 (2014), 1312.4517.

[37] H. P. Nilles, M. Srednicki, and D. Wyler, Phys.Lett. B120, 346 (1983).

[38] F. Staub (2008), 0806.0538.

[39] F. Staub, Comput.Phys.Commun. 181, 1077 (2010), 0909.2863.

[40] F. Staub, Comput.Phys.Commun. 182, 808 (2011), 1002.0840 .

[41] F. Staub, Computer Physics Communications 184, pp. 1792 (2013), 1207.0906.

[42] F. Staub (2013), 1309.7223.

[43] J. Goldstone, Nuovo Cim. 19, 154 (1961).

[44] J. E. Kim, Phys.Lett. B136, 378 (1984).

[45] H. K. Dreiner, H. E. Haber, and S. P. Martin, Phys.Rept. 494, 1 (2010), 0812.1594.

[46] T. Banks, M. Dine, and M. Graesser, Phys.Rev. D68, 075011 (2003), hep-ph/0210256.

[47] L. M. Carpenter, M. Dine, G. Festuccia, and L. Ubaldi,
Phys.Rev. D80, 125023 (2009), 0906.5015.

[48] S. Ferrara, L. Girardello, and F. Palumbo, Phys.Rev. D20, 403 (1979).

[49] S. Zheng, Eur.Phys.J. C74, 2724 (2014), 1308.5377.

[50] M. Binger and S. J. Brodsky, Phys.Rev. D69, 095007 (2004), hep-ph/0310322.

[51] J. Beringer et al. (Particle Data Group), Phys.Rev. D86, 010001 (2012).

[52] K.-Y. Choi, J. E. Kim, and L. Roszkowski, J.Korean Phys.Soc. 63, 1685 (2013), 1307.3330.

[53] A. H. Chamseddine and H. K. Dreiner, Nucl.Phys. B458, 65 (1996), hep-ph/9504337.

[54] $M_{\mathrm{SUSY}}$ is the scale of the soft terms and is typically in the $\mathrm{TeV}$ range. It should not be confused with the SUSY breaking scale $M_{\mathrm{SB}}$ which can be much higher, depending on the mechanism that mediates the SUSY breaking. For instance, one has $M_{\mathrm{SB}} \sim M_{\mathrm{GUT}}$ in gravity mediation, and $M_{\mathrm{SB}} \sim M_{\text {messenger }}$ in gauge mediation, where $M_{\text {messenger }}$ is the messenger scale.

[55] For a gauged R-symmetry see Ref. [53].

[56] We are neglecting here QCD instanton effects that generate a small axion mass. 